\title{
GENERALIZED STEENROD HOMOLOGY THEORIES
}

\author{
BY DAVID A. EDWARDS AND HAROLD M. HASTINGS
}

Communicated by P. T. Church, December 5, 1975

Introduction. Alexander duality states that for a compactum $X$ in $S^{n}$, $\check{H}^{p}(X) \cong H_{n-p-1}^{c}\left(S^{n} \backslash X\right)$, where $\check{H}^{*}$ is Čech cohomology and $H_{*}^{c}$ is ordinary homology with compact supports.

In [St] Steenrod introduced a homology theory ${ }^{S_{H}} H_{*}$ defined on the category $\mathrm{CM}$ of compact metric spaces with ${ }^{S_{H}} H_{p}(X) \cong H^{n-p-1}\left(S^{n} \backslash X\right) .{ }^{S} H_{*}$ is called ordinary Steenrod homology. Milnor [Mi1] (see [K - S]) showed that $s_{H_{*}}$ is characterized by the Eilenberg-Steenrod axioms plus two additional axioms: invariance under relative homeomorphism; and a modified form of the continuity axiom in the form of a short exact sequence relating ${ }^{s} H_{*}$ and $\check{H}_{*}$. In their recent work on extensions of $C_{*}$-algebras, Brown, Douglas and Fillmore [B - D - F] defined a functor Ext( ) on compacta whose restriction to finite complexes is $K$-homology. Kaminker and Schochet [K-S] axiomatized generalized Steenrod homology theories and showed that $\operatorname{Ext}()$ defines a generalized Steenrod homology theory on CM.

At the Operator Theory and Topology Conference (University of Georgia, April, 1975) Atiyah asked whether any generalized homology theory $h_{*}$ defined on finite complexes admits a Steenrod extension to $\mathrm{CM}$ which preserves products and operations. We announce such an extension in Theorem 2, below.

J. Kaminker recently informed us of an entirely different definition of generalized Steenrod homology theories due to D. S. Kahn, C. Schochet and himself.

Background and theorems. We will need an appropriate category and homotopy category of inverse systems of spaces. In [Gro] Grothendieck showed how to associate to any category $C$ another category pro- $C$ whose objects are inverse systems in $C$ indexed by "filtering categories" and whose morphisms are so defined as to make cofinal systems isomorphic. In [Q] Quillen introduced the notion of a model category as an axiomatization of homotopy theory on Top and SS (= the category of simplical sets).

A model category is a category $C$ together with three classes of morphisms called cofibrations, fibrations, and weak equivalences, which satisfy "the usual $55 \mathrm{H} 25$.

AMS (MOS) subject classifications (1970). Primary 46L05, 47B30, 55B15, 55B20,

Key words and phrases. Prohomotopy theory, generalized Steenrod homology theories. Copyright $\Subset$ 1976, American Mathematical Society 
properties." The homotopy category of $C, \mathrm{Ho}(C)$, is obtained from $C$ by formally inverting the weak equivalences in $C$. In $[\mathbf{E}-\mathbf{H}]$ we show how to extend a model structure on $C$ to pro- $C$.

If $h_{*}$ is a generalized homology theory defined on pointed finite complexes, then there is a $C W$-spectrum $E$ such that

$$
h_{n}(X)=\operatorname{Ho}(\mathrm{Sp})\left(S^{n}, X \wedge E\right),
$$

where $\mathrm{Ho}(\mathrm{Sp})$ is the homotopy category of $\mathrm{CW}$ spectra. Let $|\cdot|: \mathrm{SS} \rightarrow \mathrm{CW}$ be the geometric realization functor. Then we can extend $h_{*}$ to SS by the formula

$$
h_{n}(X) \equiv \operatorname{Ho}(\mathrm{Sp})\left(S^{n},\left(|X| \vee_{*}\right) \wedge E\right) \text {. }
$$

THEOREM $1[\mathbf{E}-\mathbf{H}]$. The functors

$$
s_{h_{n}}\left(\left\{X_{j}\right\}\right) \equiv \operatorname{Ho}(\text { pro-Sp })\left(S^{n},\left\{\left(\left|X_{j}\right| \vee_{*}\right) \wedge E\right\}\right)
$$

and

$$
\check{h}_{n}\left(\left\{X_{j}\right\}\right) \equiv \operatorname{pro}-H o(\mathrm{Sp})\left(S^{n},\left\{\left(\left|X_{j}\right| \vee_{*}\right) \wedge E\right\}\right)
$$

define generalized Steenrod and $\check{C}$ ech extensions of $h_{*}$ to pro-SS.

REMARKS. The axioms for Steenrod and Čech homology theories on proSS are similar to the axioms on CM.

In order to apply Theorem 1 to geometric situations, one needs to have a functor from a geometric category into pro-SS which is homotopy invariant, preserves cofibration sequences (up to homotopy) and is "continuous." The following two functors have these properties.

The end functor. Let $X$ be a locally compact ANR and let $\epsilon(X) \equiv\{X|C| C$ is a compact subset of $X$ \}. Then $\epsilon$ determines a functor from the proper category $P$ to pro-ANR which satisfies the above conditions. Hence, the compositions

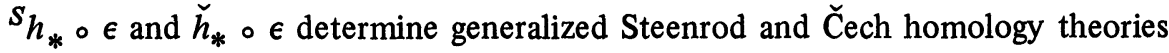
at $\infty$.

The Vietoris functor. The Vietoris nerve of an open covering $U=\{U\}$ of a space $X$ is the simplicial set $V N(U)$ in which a typical $n$-simplex is an $(n+1)$-tuple of points of $X$ all contained within an open set $U \in U$. Let $V(X)$ $=\{V N(U) \mid U$ is an open covering of $X\}$, bonded by refining maps. Then $V$ determines a functor $V: \mathrm{CM} \rightarrow$ pro-SS which satisfies the above conditions (this is technically difficult). We thus obtain the following theorem.

THEOREM $2[\mathbf{E}-\mathbf{H}]$. The functors $s_{h_{*}} \circ V$ and $\check{h}_{*} \circ V$ yield generalized Steenrod and Čech homology theories on CM.

REMARKS. We can give a geometric description of Steenrod bordism theories using infinite mapping telescopes. 
Friedlander's rigid etale functor [Fr] can probably be used to obtain a Steenrod-Etale homology theory.

\section{REFERENCES}

[B-D-F] L. Brown, R. Douglas and P. Fillmore, Unitary equivalence modulo the compact operators and extensions of $C^{*}$-algebras, Proc. Conf. on Operator Theory (Dalhousie Univ., Halifax, N. S., 1973), Lecture Notes in Math., vol. 345, Springer-Verlag, Berlin and New York, 1973, pp. 58-128.

[E-H] D. A. Edwards and H. M. Hastings, Čech and Steenrod homotopy theories with applications to geometric topology, Lecture Notes in Math., Springer-Verlag, Berlin and New York (to appear).

[Fr] E. M. Friedlander, Computations of $K$-theories of finite fields (to appear).

[Gro] A. Grothendieck, Technique de decente et théorèmes d'existence en géométrie algébrique. I. Séminaire Bourbaki $12 \mathrm{e}$ année 1959/60, Exposé 190, Secrétariat mathématique, Paris, 1960. MR 23 \#A2273.

[K-S] J. Kaminker and C. Schochet, $K$-theory and Steenrod homology: applications to the Brown-Douglas-Fillmore theory of operator algebras, Trans. Amer. Math. Soc. (to appear).

[Mil] J. Milnor, On the Steenrod homology theory, Berkely mimeo, 1961.

[Q] D. Quillen, Homotopical algebra, Lecture Notes in Math., vol. 43, SpringerVerlag, Berlin and New York, 1967. MR $36 \# 6480$.

[St] N. E. Steenrod, Regular cycles on compact metric spaces, Ann. of Math (2) 41 (1940), 833-851. MR 2, 73.

DEPARTMENT OF MATHEMATICAL SCIENCES, STATE UNIVERSITY OF NEW YORK AT BINGHAMTON, BINGHAMTON, NEW YORK 13901

DEPARTMENT OF MATHEMATICS, HOFSTRA UNIVERSITY, HEMPSTEAD, NEW YORK 11550 